\title{
METHODOLOGY TO EVALUATE THE IMPACT OF AMHS DESIGN CHARACTERISTICS ON OPERATIONAL FAB PERFORMANCE
}

\author{
Gabriel Gaxiola \\ Detlef Pabst \\ Eric Christensen \\ GLOBALFOUNDRIES U.S. Inc. \\ Malta, NY, USA
}

\author{
David Wizelman \\ Final Phase Systems, LLC \\ Austin, TX, USA
}

\begin{abstract}
Today's 300mm semiconductor facilities rely almost completely on Automated Material Handling Systems (AMHS) to transport wafers to equipment and storage areas in the wafer fabrication plant (fab). As the cost of equipment increases and the process technology becomes more and more sensitive to delivery times between steps, AMHS performance has become increasingly important to overall factory performance. Current AMHS design methods focus primarily on optimizing the balance between AMHS cost and AMHS performance. Understanding the influence of AMHS performance on fab operations has become an area of focus during the design process.

This paper proposes a methodology to correlate AMHS performance measurements with simulated fab performance measures using a linked AMHS-fab model. This methodology facilitates model setup, scenario modification, model linkage, and calculations of performance impact. A sample evaluation study demonstrates the validation and analysis process, and derives conclusions applicable during the AMHS design process.
\end{abstract}

\section{INTRODUCTION}

This paper studies the impact of the AMHS on overall fab performance using an offline linked AMHSfab model. Understanding the influence of AMHS performance on fab operations can offer additional perspective on the overall business decisions being made during the AMHS design process. Specific AMHS metrics like delivery time, waiting time, pickup averages, and percentiles are typically used to evaluate the efficiency of the AMHS. Unfortunately it is usually difficult to correlate these metrics to the real impact on manufacturing. Although it is easy to understand that lower delivery and pickup times are better for the performance of the entire fab it is not easy to understand what the real requirements should be when just looking at these metrics. Also, different equipment and areas operate in different ways, therefore the requirement for one area/equipment might be unnecessary or not enough somewhere else.

While it may be beneficial to use AMHS-fab linked models in order to make decisions when designing the AMHS, creating this kind of model and using it effectively in practice is not a straightforward task. There are many details involved in the creation of the fab model itself, the AMHS model itself, and their linkage, all which makes the creation of these models lengthy and complex. Additionally, inputs to the models are not static so there is always potential for new, changing scenarios. This paper proposes a methodology for an efficient application of these models which includes 1) the automation of the creation of a factory model for a fab that is not yet operational 2) the re-use of existing AMHS models and automation of FromTo creation and, 3) the implementation of the necessary AMHS components and useful statistics in the factory model.

The following figure (Figure 1) represents the different inputs for the models, the integration/automation process, and the outputs. For the AMHS model the inputs are the production planning inputs (process flows, starts plan, equipment lists) as well as the physical AMHS and equipment layouts 


\section{Gaxiola, Pabst, Christensen, and Wizelman}

and storage definitions. The factory model also uses the production planning inputs but these inputs are matched with real fab data to create a set of input files usable by the factory model. Additionally other information from the fab database (tool availability, dedications, holds, etc.) is also used to create the input files for the factory model. The output of the AMHS model (delivery times and pick up time logs) goes through an automation process and statistical analysis to create point to point information between all tools and storages. This process results in a FromTo table which is provided as input into the factory model along with the storage definitions. Finally, the factory model uses all this data to predict cycle time (CT), work in process (WIP), and equipment utilization including AMHS statistics.

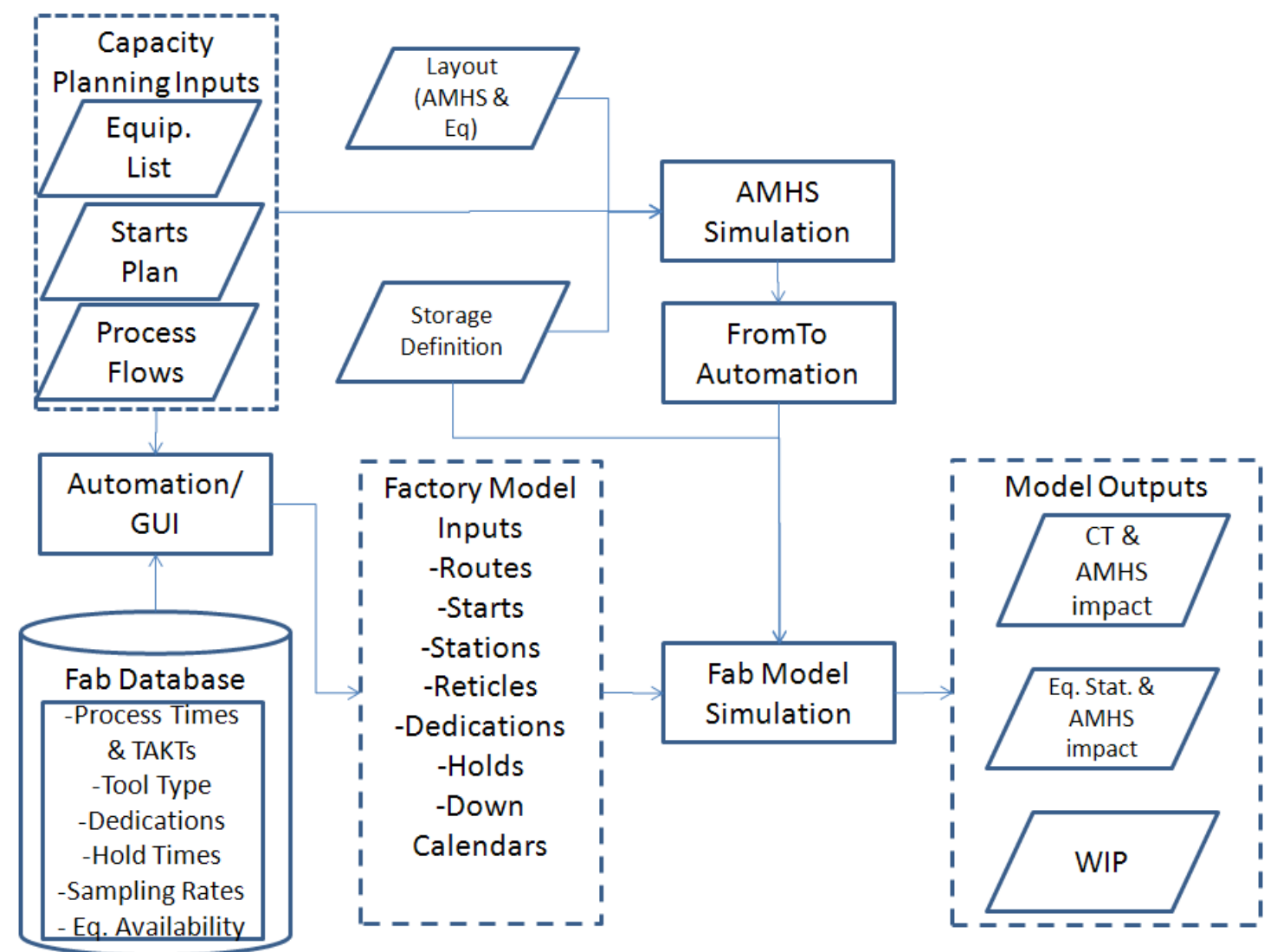

Figure 1. AMHS-Fab Model Linkage Process

The remainder of this paper is organized as follow: Section 2 is a review of related work. Section 3 describes the basic factory model prior to the addition of any AMHS components. Section 4 describes the AMHS model and the automation process to generate the "FromTo" delivery time profile. Section 5 describes how the integration process takes place within the factory model to incorporate the AMHS components and collect the necessary statistics. Section 6 shows the results of an initial application of this methodology. Finally, Section 7 summarizes conclusions and describes future work.

\section{RELATED WORK}

In literature reviewed by the authors most AMHS simulations focus on AMHS analysis without looking at direct impact on fab performance. These AMHS simulations mostly rely on throughput, vehicle count, delivery time and waiting time statistics to evaluate and compare designs. For example, Jimenez et al. (2002) looks at an AMHS design based on rails and lifters in different floors and tries to minimize average lot-delivery time. Sturm et al. (2003) outlines the planning approach for two AMHS designs used for 
interbay transportation in $200 \mathrm{~mm}$ wafer fabs with regard to good performance and robustness of the system. Nazzal and McGinnis (2006) proposes an analytical model using an extended Markov chain useful in the design of AMHS. Gaxiola et al. (2004) evaluates the benefit of using a redundant overhead shuttle (OHS) system in combination with an interconnected overhead transport (OHT) system to support automated movement of non-production wafers. Miller et al. (2011) discusses the design and test of conveyorbased AMHS configurations with emphasis on comparing centralized versus distributed storage systems. Nazzal et al. (2008) proposes an analytical model for the design of a conveyor-based AMHS to estimate the work-in-process on the conveyor and assess the system stability. Gaxiola et al. (2012) discusses different approaches for evaluating AMHS design during fab expansions.

A limited set of literature researched described usage of linked AMHS - fab models. Jimenez et al. (2010) used integrated models to evaluate the impact of Small Lot Manufacturing (SML) and to test changes in the factory logic. Kiba et al. (2009) describes an integrated model including detailed transport and production features and evaluates impact of AMHS data on vehicle watermarks determination. Kong (2007) introduces one more simulation step, the production simulation step where the production logic is implemented and the throughput of the semiconductor line can be predicted prior the AMHS simulation. Jimenez et al. (2008) identifies a method for classifying a fab model by the level of capacity detail, the level of AMHS detail, or the level of capacity/AMHS detail.

More relevant to this paper is the work from Jimenez et al. (2005) where an integrated AMHS - fab model via a "FromTo" provides similar accuracy when compared to fully integrated models. This paper extends on this simpler "FromTo" linkage methodology but applied to a more realistic case of a semiconductor fab, where models need to be created, linked and modified rapidly and need to support a high amount of input data and changing scenarios.

\section{BASIC FACTORY MODEL}

A multi-product $300 \mathrm{~mm}$ semiconductor fab simulation model serves as a base for the development and evaluation of the proposed methodology. This section describes the usual steps of model definition, automated population with data, and model validation.

\subsection{Model Definition}

The considered simulation model represents the fab design of a new GLOBALFOUNDRIES fab at full ramp and is modeled with AutoSchedAP 10.0.2.19 (64-bit). Standard AutoSchedAP features implement the basic model characteristics as described in Table 1.

A customization extends the standard AutoSchedAP features with more detailed tool processing models and dispatching logic. The deterministic tool models provide process start intervals and process durations for lots or batches of lots on tool types that combine multi-capacity and overlapping effects in different manners. This is required to achieve sufficient timing accuracy for stations like furnaces, lithography, or cluster tools. Some of these tools models require special dispatching rules that combine multiple lots into same-recipe batches (parallel) or cascades (serial) with suitable lot selection logic that ensures sufficient throughput levels and reticle availability in the simulation model.

\begin{tabular}{|l|l|l|}
\hline Model aspect & Modeled entity/logic & Non-deterministic? \\
\hline \multirow{5}{*}{ Factory } & $\begin{array}{l}\text { Stations grouped by matching type in sta- } \\
\text { tion families }\end{array}$ & - \\
\cline { 2 - 3 } & Reticles & - \\
\cline { 2 - 3 } & $\begin{array}{l}\text { Probabilistic down times for stations (down } \\
\text { calendars) }\end{array}$ & Yes \\
\hline Product definitions & $\begin{array}{l}\text { Parts and routes (sequence of operations that } \\
\text { a part needs to complete) }\end{array}$ & - \\
\hline
\end{tabular}


Gaxiola, Pabst, Christensen, and Wizelman

\begin{tabular}{|l|l|l|}
\hline \multirow{5}{*}{} & $\begin{array}{l}\text { Subsets of stations within station families } \\
\text { that are qualified to process this product at a } \\
\text { certain operation (so called dedications) }\end{array}$ & - \\
\cline { 2 - 3 } & Process control sampling probabilities & Yes \\
\cline { 2 - 3 } & Lot hold probabilities & Yes \\
\hline \multirow{2}{*}{ Loading } & Lot start distribution & Yes \\
\cline { 2 - 3 } & Multi-year simulation horizon & - \\
\hline
\end{tabular}

Table 1. Basic Simulation Model Characteristics

\subsection{Automatic Model Population}

Simulation models are derived from a description of the fab entities with their current state and of timing information for future state transitions. Factory control systems contain a representation of the current qualified and dedicated tool sets, reticles, active routes, work in progress, planned lot starts, and PM calendars. Such systems also trace fab state change events and update statistics for process durations, process start intervals, time-to-failures, time-to-repairs, sampling rates, lot hold rates and hold durations. At GLOBALFOUNDRIES, an automated procedure accesses both of these data sources to populate simulation models that are able to project forward from the current fab state.

In order to generate simulation models that represent the full-build-out state of a new ramping fab, this procedure had to be adjusted. The fab capacity plan and the technology plan provide required information about planned tools, reticles, and routes. However, no fab observations to infer the required fullramp timing statistics are available. Individual statistics derived from another full-ramp fab need to be used as reference (as listed in the "Fab Database" of Figure 1 above). An automated pattern matching algorithm can identify similarities in the tool type description and/or process descriptions of the full-ramp fab and the ramping fab and thereby populate the new fab's simulation model with timing data.

\subsection{Model Validation}

The final step of the model creation validates the ability of the model to reasonably predict the station throughput and lot cycle time impact due to the AMHS design. This should be done at least on the three levels of steady-state average product cycle times, overall throughput and the individual tool utilization levels. In the case that no real-fab observations are available, as for the here considered new ramping fab, the capacity and fab planning models need to provide the reference values to validate against.

The iterative simulation modeling process typically generates initial models that underestimate the expected variability of the real fab and therefore yields product cycle times and overall throughput rates that lay below the acceptable levels. This drives the extension of the model with variability increasing features like the above mentioned reticle considerations and tool dedication schemes or the timing adjustment of flow disruptions like tool down time distributions, sampling delays, or hold delays.

Comparisons of simulation observations for individual tool utilizations with reference values should be focused on the heavy utilized station families, as those clearly are expected to have the largest influence on the intended simulation study have a low expected inaccuracy in the reference values (if not from real-fab observations). The attempt to completely match all individual tool utilizations with the reference values would need to be achived by adding a level of model details that seems unnecessary for the here intended predictive capability of the model, yet would slow down the simulation runtime. Failures of the high tool utilization observations in the simulation model to match with the reference values, or vice versa need to be addressed by adjustments to AutoSchedAP customizations for tool model and/or dispatching logic or by adjustments to the "effective" processing times. 
Gaxiola, Pabst, Christensen, and Wizelman

\section{AMHS MODEL}

AMHS models are typically built for or by GLOBALFOUNDRIES during initial AMHS design process to measure delivery times and waiting times, evaluate throughput and estimate vehicle count. This paper proposes reusing existing models to collect the raw data needed, followed by the usage of an automation process to convert the AMHS model output into a "FromTo" that can be used by the factory model.

\subsection{AMHS Model Definition}

The AMHS model used for this paper was built in AutoMod 12.4 (64-bit) and represented the AMHS design of one of GLOBALFOUNDRIES fabs at full ramp. Since the fab represented was a future fab the input requirement for the model were based on planning information such as projected process flows, start rates and equipment lists. Planned storage locations (stockers and zero footprint storages) and the AMHS layout were also used to build this model. Figure 2 represents the AMHS layout of a typical bay. Process flows were translated into point to point move requirements using a $1.8 \mathrm{x}$ safety factor to account for variability in the flows and for non-production wafer (NPW) movement. Lots were created in the originating tool or storage and destroyed in the destination tool or storage after statistics collection. The model used depicted very closely the logic of the real AMHS since it was built by an AMHS supplier with most of the similar features that will be used in a real fab AMHS, such as vehicle watermarks, push/pull logic, vehicle reassignments, etc. The initial model used 610 carrier vehicles in order to reach GLOBALFOUNDIRES throughput, lot size and delivery time requirements. For subsequent scenario analysis the original model will be changed to represent an increased fab load or a different count or distribution of vehicles in the fab, a new FromTo will be generated and added to the fab model.

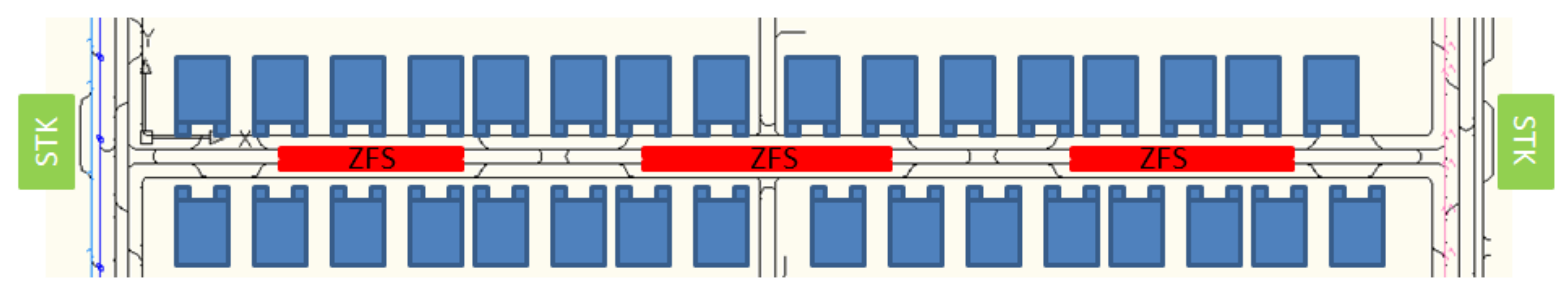

Figure 2. AMHS Typical Bay Layout

\subsection{FromTo Creation Process}

The FromTo automation tool consisted of several scripts files put together. AutoMod simulations of the AMHS generated a list of executed transports during the simulation runs. Among others it includes information about starting control point, destination control point, waiting time for retrieving vehicle and transport time to destination.

The transport data was statistically analyzed by origin equipment, origin bay and origin lane for waiting times and by equipment to equipment relations, lane to lane relations, and bay to bay relations for delivery times. In this analysis a lane was defined as a quadrant of the entire bay.

Based on a mapping list of real tool IDs to their equipment group and equipment number in the simulation and a manual list of additional equipment (including stockers and Zero Footprint Groups -ZFGs-) a list of equipments was created. For each equipment to equipment relation a parameter set for distributions of waiting time and transport time was created. The distributions were selected as detailed as possible depending on available data. For waiting times this means the waiting time distribution of the equipment was selected if a significant number of transports had been executed from this equipment. Otherwise the time distribution of the lane was selected if there were a significant number of transports observed from this lane. Otherwise the time distribution of the whole bay of the equipment was selected. If this still did not show sufficient transport observation counts the distribution of all waiting times was selected. Similar hierarchies were created for transport times relations. 
A comparison of fitted gamma and lognormal distributions for a few cases showed a very similar approximation of both The lognormal distribution was chosen however due its easier implementation (e.g. gamma distribution besides parameters alpha and beta also needed a shift of the function to get best results).

\section{FACTORY MODEL - AMHS MODEL LINKAGE}

The wafer fab process equipment simulation model, referred to as the factory model, used for this paper is described in section 3. Planned tool and storage locations were based on the layout depicted in Figure 2. For the purposes of this study, it was determined that the impact analysis desired could be achieved by using the detailed FromTo file described in Section 4.2 "FromTo Creation Process" in the factory model. Performing the model linkage in this offline manner simplifies model creation, maintenance, and troubleshooting as compared to an online linked model where the factory model and AMHS model are communicating real-time during simulation runs.

\subsection{Storage Definition}

Although the tool and storage locations were based on the layout depicted in Figure 2, the granularity of the storage locations used in the factory model was generalized to the bay and bay/toolset levels only since move times from different locations within a bay were deemed insignificant. Each bay represents a rectangular section of the layout outlined by AMHS overhead track. The storage model used has three tiers of storages, defined below in order of proximity to the tools.

Station Storages Equipment load ports (LP) and internal buffers for storage at the tool (Station)

Stage Storages Side and under-track storages which are grouped together into zero footprint groups (ZFG) for storage near groups of tools running similar processes (Stnfams)

Stnfam Storages Stockers (STK) assigned to each Stnfam for general or longer term storage within each bay

The Station Storages were defined, and their capacities set, based on actual tool configuration. The Stage Storages were defined at the bay/toolset level so that each toolset which has tools in a given bay has a corresponding ZFG dedicated to storage of its material. The capacity of the ZFG's were set based on a proportion of the total ZFG capacity available in the bay to the throughput of each toolset in the bay. Finally, each bay was assigned a single stocker and this stocker was set as the Stnfam Storage for each toolset in each bay. The capacity of each stocker was considered infinite. AutoSchedAP's bumping logic was used to push carriers to an appropriate Stage or Stnfam Storage in cases where a tool was not ready to select a lot from the carrier immediately after it finished processing at the prior step. If a tool was ready to select a lot, then the tool pulled the carrier to its Station Storage immediately. In this scenario, it is possible for a lot to be selected and the Station Storage to be full. Default AutoSchedAP behavior will cause this carrier to be moved to the first available storage in the storage list so a customization was created to move carriers in this situation to the appropriate ZFG, if available, or stocker and wait until the "blocking" carrier was retrieved. This blocking scenario is described further in Section 5.3.

In terms of AutoSchedAP configuration, an example station and related storage and bumping configuration is provided below in Table 2 and Figure 3.

\begin{tabular}{|l|l|l|l|l|l|}
\hline STNFAM & STN & STNSTOR & STAGESTOR & STNFAMSTOR & BumpToStorageTime \\
\hline PolishOx & PolOx01 & LP_PolOx01 & ZFG_PolishOx_Bay02 & STK_Bay02 & 0 \\
\hline PolishOx & PolOx02 & LP_PolOx02 & ZFG_PolishOx_Bay02 & & 0 \\
\hline
\end{tabular}


Gaxiola, Pabst, Christensen, and Wizelman

\begin{tabular}{|l|l|l|l|l|l|}
\hline PolishOx & PolOx03 & LP_PolOx03 & ZFG_PolishOx_Bay02 & & 0 \\
\hline PolishOx & PolOx04 & LP_PolOx04 & ZFG_PolishOx_Bay02 & & 0 \\
\hline PolishInspect & PolInsp01 & LP_PolInsp01 & ZFG_PolishInspect_Bay02 & STK_Bay02 & 0 \\
\hline PolishInspect & PolInsp02 & LP_PolInsp02 & ZFG_PolishInspect_Bay02 & & 0 \\
\hline
\end{tabular}

Table 2. AutoSchedAP Station Configuration

(LP - Load Ports, ZFG - Zero Footprint Group Track Storage, STK - Stocker)

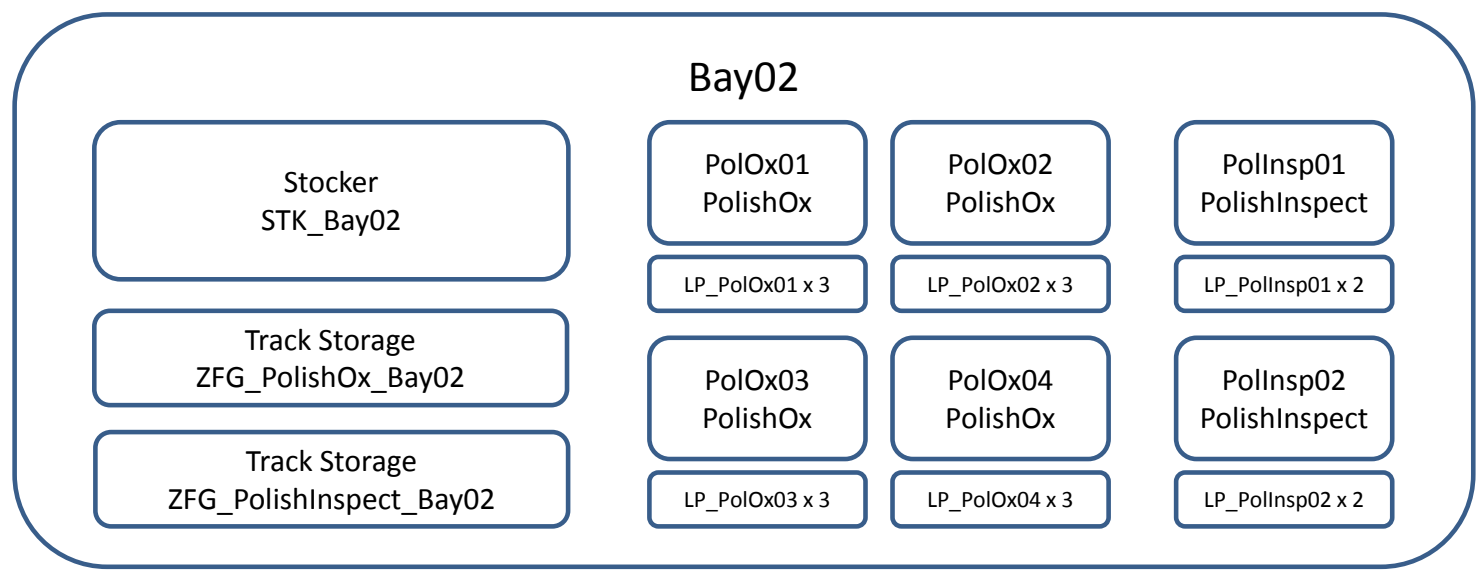

Figure 3. Bay with Stocker, Track Storages, and Tools

\subsection{Transports FromTo Definition}

The FromTo input described in Section 4.2 was adapted to match the specific naming schema required in the AutoSchedAP model. For example, ZFG_Bay02 was split into ZFG_PolishOx_Bay02 and ZFG_PolishInspect_Bay02, although in both cases the transport and retrieval information was obtained from the ZFG_Bay02 data set.

In terms of AutoSchedAP configuration, an example FromTo configuration is provided below in Table 3 .

\begin{tabular}{|l|l|r|r|l|l|r|r|l|l|}
\hline FROMLOC & TOLOC & RTIME & RTIME2 & RDIS T & RUNITS & DTIME & DTIME2 & DDIST & DUNITS \\
\hline LP_PolOx01 & LP_PolInsp01 & 3 & 0.5 & lognormal & min & 6 & 0.8 & $\operatorname{lognormal}$ & min \\
\hline LP_PolOx01 & ZFG_PolishInspect_Bay02 & 3 & 0.5 & lognormal & min & 3 & 0.5 & $\operatorname{lognormal}$ & min \\
\hline LP_PolOx01 & STK_Bay02 & 3 & 0.5 & lognormal & min & 4 & 0.6 & $\operatorname{lognormal}$ & min \\
\hline ZFG_PolishOx_Bay02 & LP_PolOx01 & 3 & 0.5 & lognormal & min & 3 & 0.5 & $\operatorname{lognormal}$ & min \\
\hline STK_Bay02 & LP_PolOx01 & 3 & 0.5 & lognormal & min & 4 & 0.6 & lognormal & min \\
\hline
\end{tabular}

Table 3. AutoSchedAP FromTo Configuration

\subsection{AMHS Statistics Definition}

AutoSchedAP provides a standard set of tool and lot state and transport statistics which are useful to understand many elements of the impact of the AMHS's delivery time profile on fab performance. By comparing one factory model to another using different AMHS FromTo delivery profiles, including a profile where all delivery times are zero, many conclusions can be drawn about the AMHS's impact on fab performance in terms of utilization, cycle time, output, and inventory levels. However, detailed AMHS delivery related information, including the concept of AMHS "blocking," is not covered by this type of analysis. Blocking refers to the effect observed when a tool is ready to process a lot but that lot cannot be delivered to the tool because an existing carrier is occupying storage capacity that is needed for the new 
carrier. This happens when the occupying carrier has not yet been retrieved and usually, unless the carrier is allowed to wait in its current storage (this is configurable in the AutoSchedAP bumping logic configuration), causes the lot that is to be processed next to be transported to a ZFG or stocker rather than directly to the tool. Since re-routing was not allowed in this model the carrier would travel to the ZFG or stocker and either move again immediately to the tool if the blocking lot had been retrieved, or wait in the ZFG or stocker until the blocking lot was retrieved and then move to the tool.

Figure 4 shows the standard move scenarios that were under observation. Moves to and from stockers are not depicted since they are essentially the same as moves to and from ZFG's, albeit usually with slightly higher delivery times.

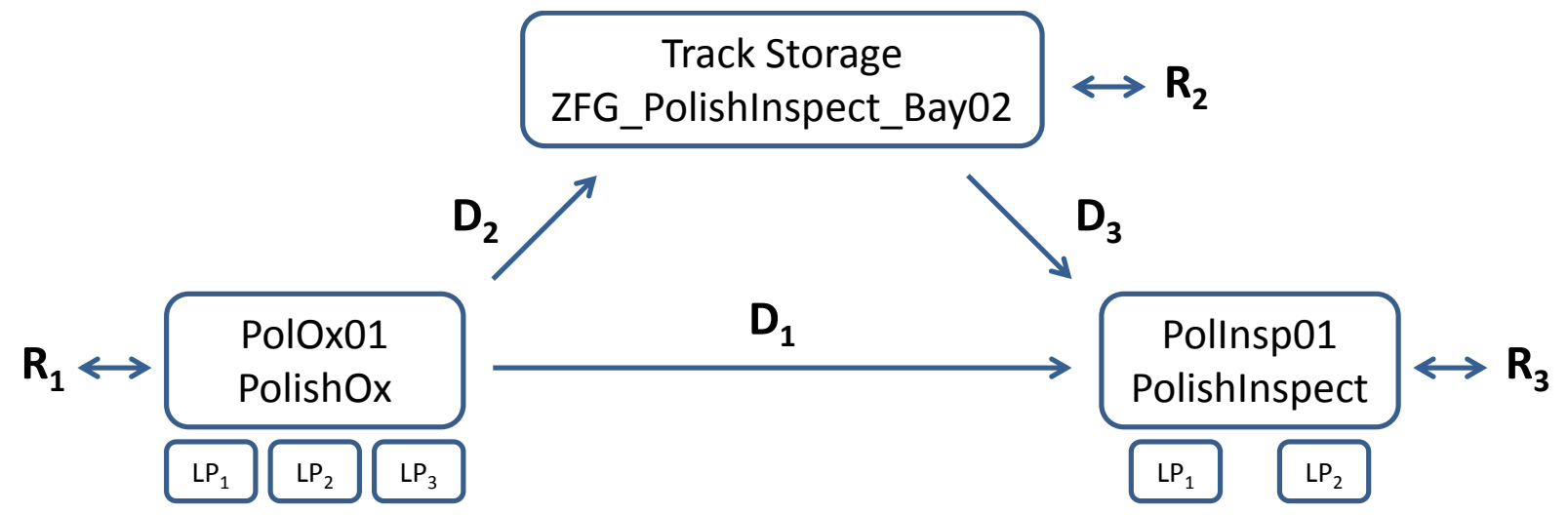

Figure 4. Move Scenarios from Tool PolOx01 to Tool PolInsp01 (R Denotes Retrieval and D Denotes Delivery)

Three statistics were introduced to help understand the amount of time carriers spent being transported and delays associated with transport due to blocking conditions. An AutoSchedAP customization was created to record these statistics. These statistics were added to the lot and part reports.

TravelToStn The total duration required for the carrier to travel from its prior station to the next station on which its lot(s) will process. Includes all AMHS retrieval and travel time, including time being delivered to and retrieved from intermediate storages between the stations.

TravelToStnDelay The part of the duration TravelToStn during which the next station is ready to start processing the lot and one of the following scenarios takes place:

- The carrier is on the load port of the current station and has to be retrieved and travel to the next station In this case TravelToStnDelay $=$ TravelToStn.

- The carrier is in the process of being retrieved or is traveling to the $\mathrm{ZFG} / \mathrm{STK}$ of the next station, or is waiting in this ZFG/STK. In this case TravelToStnDelay $<$ TravelToStn.

BlockingTime Duration during which the next station is ready to start processing the lot but it has to wait in the ZFG/STK because a completed lot is still on the next station's load port waiting to be retrieved.

The following scenarios were comprehended and corresponding calculations made to obtain these statistics. Figure 4 shows the move options that are referenced in these scenarios. 
Gaxiola, Pabst, Christensen, and Wizelman

\begin{tabular}{|c|c|}
\hline SCENARIO & CALCULATIONS \\
\hline $\begin{array}{l}\text { LotX on PolOx01 } \mathrm{LP}_{1} \text { is delivered to PolInsp01 } \mathrm{LP}_{1} \text { that is } \\
\text { free. }\end{array}$ & TravelToStn $=$ TravelToStnDelay $=R_{1}+D_{1}$ \\
\hline $\begin{array}{l}\text { LotX in ZFG_PolishInspect_Bay02 is delivered to } \\
\text { PolInsp01 LP } \text { that is free. }\end{array}$ & $\begin{array}{l}\text { TravelToStn }=R_{1}+D_{2}+R_{2}+D_{3} \\
\text { TravelToStnDelay }=R_{2}+D_{3}\end{array}$ \\
\hline $\begin{array}{l}\text { LotX on PolOx01 is selected by PolInsp01 but } \mathrm{LP}_{1} \text { and } \mathrm{LP}_{2} \\
\text { are both occupied with lots. One of these lots has already } \\
\text { completed and is waiting to be retrieved (blocking). LotX } \\
\text { will be delivered to ZFG_PolishInspect_Bay02 and then } \\
\text { PolInsp01 when a port becomes free. }\end{array}$ & $\begin{array}{l}\text { TravelToStn }=\text { TravelToStnDelay }=\mathrm{R}_{1}+\mathrm{D}_{2}+\mathrm{R}_{2}+\mathrm{D}_{3} \\
\text { BlockingTime }=\max \left(0, \mathrm{~W}_{R 3} * \mathrm{R}_{3}-\left(\mathrm{R}_{1}+\mathrm{D}_{2}\right)\right) \text { where } \mathrm{W}_{\mathrm{R} 3} \text { ranges } \\
\text { from } 0-1 \text { scaling } \mathrm{R}_{3} \text { to the remaining retrieval time. This blocking } \\
\text { time consists of the potential wait time in the } \mathrm{ZFG} \text { after the lot } \\
\text { arrived there and until the LP becomes free. }\end{array}$ \\
\hline $\begin{array}{l}\text { LotX is in the process of being retrieved or is traveling } \\
\text { from its last station PolOx01 to the ZFG/STK of the next } \\
\text { station at the time it is selected by PolInsp01 but } \mathrm{LP}_{1} \text { and } \\
\mathrm{LP}_{2} \text { are both occupied with lots. One of these lots has al- } \\
\text { ready completed and is waiting to be retrieved (blocking). } \\
\text { LotX will be delivered to ZFG_PolishInspect_Bay02 and } \\
\text { then PolInsp01 when a port becomes free. }\end{array}$ & $\begin{array}{l}\text { TravelToStn }=\mathrm{R} 1+\mathrm{D} 2+\mathrm{R} 2+\mathrm{D} 3 \\
\text { TravelToStnDelay }=\mathrm{R} 2+\mathrm{D} 3 \\
\text { BlockingTime }=\max (0, \mathrm{WR} 3 * \mathrm{R} 3-(\mathrm{WR} 1 * \mathrm{R} 1+\mathrm{WD} 2 * \mathrm{D} 2)) \\
\text { where where WR } 1, \mathrm{WR} 3, \mathrm{WD} 2 \text {, range from } 0-1 \text { and scale } \mathrm{R} 1 \text {, } \\
\mathrm{R} 3 \text { and } \mathrm{D} 2 \text {, respectively to their remaining time.. The blocking } \\
\text { time consists only of a potential wait time in the ZFG after the lot } \\
\text { arrives there and until the LP becomes free. The LP blocking sit- } \\
\text { uation did not cause the ZFG detour of the approaching lot in this } \\
\text { case. }\end{array}$ \\
\hline $\begin{array}{l}\text { LotX in ZFG_PolishInspect_Bay02 is selected by } \\
\text { PolInsp01 but } \mathrm{LP}_{1} \text { and } \mathrm{LP}_{2} \text { are both occupied with lots. } \\
\text { One of these lots has already completed and is waiting to } \\
\text { be retrieved (blocking). LotX will be delivered to } \\
\text { PolInsp01 when a port becomes free. }\end{array}$ & $\begin{array}{l}\text { TravelToStn }=\mathrm{R} 1+\mathrm{D} 2+\mathrm{R} 2+\mathrm{D} 3 \\
\text { TravelToStnDelay }=\mathrm{R} 2+\mathrm{D} 3 \\
\text { BlockingTime }=\mathrm{WR} 3 * \mathrm{R} 3 \text { where WR3 ranges from } 0-1 \text { scaling } \\
\mathrm{R} 3 \text { to the remaining retrieval time. }\end{array}$ \\
\hline
\end{tabular}

Table 4. Move Scenarios and Corresponding Calculations

A customization was also created to record tool blocking related statistics. Relevant standard AutoSchedAP tool states were given an analogous blocking state, which is a subset of the standard state. These statistics were added to the station and station family reports. The blocking states created were:

\%LotsBlocked

BLOCKING-PROC\%

BLOCKING-WTTRAN\%

BLOCKING-DOWN\%

BLOCKING-WTSTN\%

BLOCKING-TRAN\%
Percentage of carriers that were blocked, regardless of duration.

Processing - the tool is still processing a lot and a blocking conditions exists for the next lot it has chosen

Waiting for retrieval - the tool is ready to process the next lot and is waiting for the carrier to be picked up while a blocking condition exists for that carrier

Down - the tool has gone down since choosing its next lot and a blocking condition exists for the carrier

Waiting for station - the next lot has all non-tool resources ready and but is waiting for the tool and a blocking condition exists

In transit - the tool is waiting for its next carrier to be delivered after it was retrieved and the blocking conditions exists

Note that BLOCKING-IDLE\% is not a state that was used. This is because in order for a blocking situation to exist, a lot must already be selected for the tool and therefore the tool's state during that time, unless still processing or completing another lot, will be either WTRAN or TRAN.

By combining the standard AutoSchedAP statistics with these customized AMHS statistics, a complete picture of AMHS impact on fab performance can be drawn. 
Gaxiola, Pabst, Christensen, and Wizelman

\section{EVALUATION STUDY RESULTS}

An initial study was made to compare the current AMHS design parameters with a hypothetical situation with no AMHS delays. This approach enabled better understanding of the impact of the AMHS on the overall system as well as validation of the newly implemented transport statistics. The model was run for a duration of four years and since the model didn't include initial WIP, the first year of statistics was discarded to account for the warm up period. Results showed in this section represent the average or sum of 1561 -week snaps.

\subsection{Baseline Results}

Table 5 shows the results for throughput, transport time statistics and CT deltas for scenarios with AMHS delays and no AMHS delays. By looking at the lot starts and lots comps columns we can see the model met the throughput requirement of about 60,000 wafers outs per month. AMHS total travel accounted for about $3.4 \%$ of total CT. More interesting for this study is the AMHS delay computation which shows the time lots were delayed for processing (e.g. next station was ready to process and lot was still traveling) was of about $1.5 \%$ of total CT. This is the time where the authors find opportunities for optimization as this time is lost time in process equipment related directly to AMHS travel. We can also see that the blocking delay was negligible. This indicates completed lots were removed quickly from the Load Ports therefore rarely causing blocking conditions. With different vehicles scenarios though this is an important statistic to understand.

From comparing the results with AMHS vs. No AMHS scenarios it is possible to observe the total impact of the AMHS on CT of about $5.3 \%$ of total CT, which could be broken down in $3.4 \%$ of total CT of direct AMHS travel plus about $1.9 \%$ of total CT of indirect impact. This indirect impact can be attributed to larger average queuing time due to lower tool availability and higher arrival process variability due to the stochastic transport delays between the station families.

\begin{tabular}{|l|c|c|c|c|c|c|}
\hline & & LOT & $\begin{array}{c}\text { TRAVEL TO } \\
\text { STN TOT } \\
\text { With AMHS }\end{array}$ & $\begin{array}{c}\text { TRAVEL TO } \\
\text { STN DELAY } \\
\text { (\% of CT) }\end{array}$ & $\begin{array}{c}\text { BLOCKING } \\
\text { TIMETOT } \\
\text { (\% of CT) }\end{array}$ & $\begin{array}{c}\text { AMHS vs. } \\
\text { No AMHS } \\
\text { Delta (\% of } \\
\text { CT) }\end{array}$ \\
\hline PART_GROUP_A & 13175 & 13168 & $3.5 \%$ & $1.6 \%$ & $0.0 \%$ & $5.8 \%$ \\
\hline PART_GROUP_B & 23250 & 23078 & $3.8 \%$ & $1.9 \%$ & $0.0 \%$ & $5.1 \%$ \\
\hline PART_GROUP_C & 6975 & 7136 & $2.7 \%$ & $1.1 \%$ & $0.0 \%$ & $3.6 \%$ \\
\hline PART_GROUP_D & 3875 & 3946 & $2.2 \%$ & $0.9 \%$ & $0.0 \%$ & $0.7 \%$ \\
\hline PART_GROUP_E & 41075 & 41479 & $3.4 \%$ & $1.5 \%$ & $0.0 \%$ & $6.7 \%$ \\
\hline Grand Total & $\mathbf{8 8 3 5 0}$ & $\mathbf{8 8 8 0 7}$ & $\mathbf{3 . 4 \%}$ & $\mathbf{1 . 5 \%}$ & $\mathbf{0 . 0 \%}$ & $\mathbf{5 . 3 \%}$ \\
\hline
\end{tabular}

Table 5 -Part Statistics - AMHS vs. No AMHS

Table 6 shows the results of the station family statistics. Standard AutoSchedAP statistics show that about $2.6 \%$ of all the stations time is in either transport or waiting for transport (\%Tran or WTran\%) states, and this number goes up to $3.7 \%$ in the bottleneck stations. However in this example in the fab, the bottleneck tools have internal buffers integrated to guarantee continual processing. Hence the AMHS statistics shown here don't reflect the actual impact of AMHS on these tools, rather these values should be interpreted as the potential AMHS effect if no internal buffers solution were in use. Also, as we saw in the step statistics, the blocking $\%$ is very low at $0.1 \%$ of the total of all stations time. 
Gaxiola, Pabst, Christensen, and Wizelman

\begin{tabular}{|l|c|c|c|c|}
\hline \multirow{2}{*}{ Row Labels } & \multicolumn{2}{|c|}{ With_AMHS } & \multicolumn{2}{c|}{ Without_AMHS } \\
\cline { 2 - 5 } & $\begin{array}{c}\text { ALL } \\
\text { StnFam }\end{array}$ & $\begin{array}{c}\text { Bottleneck } \\
\text { StnFam }\end{array}$ & $\begin{array}{c}\text { ALL } \\
\text { StnFam }\end{array}$ & $\begin{array}{c}\text { Bottleneck } \\
\text { StnFam }\end{array}$ \\
\hline Average of PROC\% & 32.9 & 71.8 & 32.9 & 71.3 \\
\hline Average of DOWN\% & 23.3 & 20.8 & 23.3 & 20.8 \\
\hline Average of WTGENRES\% & 0.0 & 0.0 & 0.0 & 0.0 \\
\hline Average of IDLE\% & 41.1 & 3.7 & 43.8 & 7.8 \\
\hline Average of TRAN\% & 1.9 & 2.8 & 0.0 & 0.0 \\
\hline Average of WTTRAN\% & 0.7 & 0.9 & 0.0 & 0.0 \\
\hline Average of WTOTHER\% & 0.0 & 0.0 & 0.0 & 0.0 \\
\hline & & & & \\
\hline Average of FamBLOCKING-LOTS\% & 0.3 & 0.0 & 0.0 & 0.0 \\
\hline Average of FamBLOCKING\% & 0.1 & 0.0 & 0.0 & 0.0 \\
\hline
\end{tabular}

Table 6 - Station Family Statistics - AMHS vs. No AMHS

\section{CONCLUSIONS AND NEXT WORK}

The methodology presented in this paper is helping GLOBALFOUNDRIES team to better understand initial requirements for AMHS and evaluate where potential savings and/or improvement could be realized. For example, a reduction of just 20 vehicles could represent saving for the company of $\$ 1$ million or more. Furthermore, a simple example presented here showed that lots are late for processing a total of 1.5 $\%$ of total CT while traveling. Due to the fact that tools often start with zero WIP, it will be impossible to completely eliminate this time but further study could give light to where improvement is possible. A vehicle sensitivity analysis and evaluation of new delivery times and pick up times profiles scenarios will be performed as one of the next steps. After having applied this methodology to design the AMHS layout and vehicle counts in support of a certain fab performance at a planned fab load, a degraded AMHS delivery FromTo profile will allow determination of the change in fab performance and the impact of the same AMHS layout at an increased fab load.

The investigated scenarios indicated that additional insight about the AMHS impact on fab performance measures could be revealed by a more detailed modeling of the orchestration between lot transports, buffer-loading times, and tool reservation times. Such additional modeling logic could include more sophisticated vehicle rerouting at tool release times and lot pre-staging strategies.

\section{REFERENCES}

Gaxiola, G., Christensen, E., Hammel, C., Stachura, P. 2012. Methodology to Best Extend AMHS for Site Expansion. 2012. In Proceedings of the 2012 Winter Simulation Conference.

Gaxiola, G., Hennessy, L., Glüer, D., Stachura, P., Christensen, E. 2004. Evaluation of an Overhead System as an Alternate Loop to an Interconnected Hoist System. 2004 Brooks Worldwide Automation Symposium, (no page numbers).

Jimenez, J., Kim, B., Fowler, J., Mackulak, G., Choung, Y and Kim, D. 2002. Operational Modeling and Simulation of an Inter-Bay AMHS in Semiconductor Wafer Fabrication. In Proceedings of the 2002 Winter Simulation Conference, 1377-1382.

Jimenez, J., Mackulak, G. and Fowler, J. 2005. Efficient Simulations for Capacity Analysis and Automated Material Handling System Design in Semiconductor Wafer Fabs. In Proceedings of the 2005 Winter Simulation Conference, 2157-2161. 
Jimenez, J., Mackulak, G. and Fowler, J. 2008. Levels of Capacity and Material Handling System Modeling for Factory Integration Decision Making in Semiconductor Wafer Fabs. IEEE Transactions on Semiconductor Manufacturing, Vol. 21, No. 4, November 2008, 600-613.

Jimenez, J., Bell, M., Adikaram, Ch., Davila,V., Wright, R and Grosser, A. 2010. AMHS Factors Enabling Small Wafer Lot Manufacturing in Semiconductor Wafer Fabs. In Proceedings of the 2010 Winter Simulation Conference, 2575-2585.

Kiba, J., Lamiable, G., Dauz'ere-P'er'es, S., and Yugma, C. 2009. Simulation of a Full 300mm Semiconductor Manufacturing Plant with Material Handling Constraints. In Proceedings of the 2009 Winter Simulation Conference, 1601-1609.

Kong, S. H. 2007. Two-step Simulation Method for Automatic Material Handling System of Semiconductor Fab. Robotics and Computer-Integrated Manufacturing 23 (2007), 409-420.

Miller, L., Bradley, A., Tish, A., Jin, T., Jimenez, J. and Wright, R. 211. Simulating Conveyor-Based AMHS Layout Configurations in Small Wafer Lot Manufacturing Environments. In Proceedings of the 2011 Winter Simulation Conference, 1944-1952.

Nazzal, D. and McGinnis, L. 2006. An Analytical Model of Vehicle-Based Automated Material Handling Systems in Semiconductor Fabs. In Proceedings of the 2006 Winter Simulation Conference, 18711879.

Nazzal, D., Carlo H., Johnson, A. and Jimenez, J. 2008. An Analytical Model for Conveyor Based AMHS in Semiconductor Wafer Fabs. In Proceedings of the 2008 Winter Simulation Conference, 2148-2155.

Sturm, R., Seidelmann, J., Dorner, J. and Reddig, K. 2003. An Approach to Robust Layout Planning of AMHS. In Proceedings of the 2003 Winter Simulation Conference, 1366-1372.

\section{AUTHOR BIOGRAPHIES}

GABRIEL GAXIOLA is a member of GLOBALFOUNDRIES' Manufacturing Technology organization where he leads the modeling and simulation activities. He has a combined experience of 15 years in consulting and semiconductor industry. In the past he has been part of Qimonda, CH2M-Hill and Brooks (PRI) Automation teams. He has a MS degree in Industrial Engineering from Arizona State University and his e-mail address is gabriel.gaxiola@GLOBALFOUNDRIES.com.

DETLEF PABST is a member of GLOBALFOUNDRIES' Manufacturing Technology organization where he leads the design and development of global WIP Flow Optimization solutions for the company. He has a combined experience of 10 years of semiconductor manufacturing related work. Detlef holds graduate degrees in Mathematics and Informatics from the University of Wuerzburg, Germany and can be contacted via email@ detlef.pabst@GLOBALFOUNDRIES.com.

ERIC CHRISTENSEN is a member of GLOBALFOUNDRIES' Manufacturing Technology organization where he leads the integration and development of Automation Technology solutions for the company. He has a combined 26 years of semiconductor manufacturing systems experience at AMD and GLOBALFOUNDRIES including a 2 year assignment at SEMATECH. Eric has a BS degree in Mechanical Engineering from the University of Texas and can be contacted via email @ eric.christensen@GLOBALFOUNDRIES.com.

DAVID WIZELMAN is President and co-founder of Final Phase Systems, a nanotechnology manufacturing software and services provider focused on improving operational efficiency through dispatching, scheduling, reporting, and related Industrial Engineering systems and analysis. He has 14 years of experience working in the semiconductor industry and prior to starting Final Phase Systems he was the manager of the Decision Support Systems group at AMD and then Spansion's Fab25 in Austin, TX. He holds a BS in Industrial Engineering and Operations Research from the University of California at Berkeley and can be reached at david.wizelman@finalphasesystems.com. 